\title{
Versorgung eines Damm- und Scheidenrisses
}

\author{
Eine Gynäkologin musste sich vor dem Landgericht Bonn verantworten. Ihr wurde vorgeworfen, einen \\ Damm- und Scheidenriss ihrer Patientin falsch versorgt zu haben.
}

B ei der Geburt ihres Kindes erlitt die Patientin einen Dammriss II. Grades sowie einen Scheidenriss bis ins mittlere Drittel der Scheide. Die Schnitte wurden nach Abschluss des Geburtsvorgangs von der Gynäkologin genäht. Vier Tage später wurde die Patientin nach einer Abschlussuntersuchung durch die Gynäkologin aus der stationären Behandlung entlassen. Die Dokumentation war unauffällig, ebenso die Dokumentation der Hebamme im Rahmen der häuslichen Nachsorge. Bei einer späteren Nachuntersuchung beschrieb ein niedergelassener Kollege den Damm als „stark häutig hochgezogen“. In weiterer Folge unterzog sich die Patientin einer operativen Revision.

Die Patientin warf der Gynäkologin vor, ihren Scheideneingang „nahezu zugenäht" zu haben. Deswegen sei ein neuerlicher Eingriff erforderlich geworden. Aufgrund eines Behandlungsfehlers der Gynäkologin seien ihr bis heute Empfindungsstörungen im Bereich der Scheide verblieben. Ihr Sexualleben sei erheblich beeinträchtigt.

\section{So sah das Gericht den Fall}

Das Landgericht Bonn hat - sachverständig beraten - einen Sorgfaltspflichtverstoß der Gynäkologin verneint (Urteil vom 29.06.2012, Az. 9 O 125/11). Die unter der Geburt eingetretenen Verletzungen seien nicht immer zu verhindern. Vernarbungen nach Geburtsverletzungen seien häufig und oftmals unabhängig von der Art der Nahtqualität. Sie entstünden häufig durch Verklebungen während der Wundheilungsphase. Aus der Dokumentation ergebe sich kein Anhalt für ein sorgfaltswidriges Vorgehen.
Aus der Beschreibung des niedergelassenen Kollegen sei zu folgern, dass das Muskelgewebe ordnungsgemäß verheilt und lediglich die Haut zu eng zusammengewachsen sei. Darüber hinaus sei auch eine Speculumeinstellung beschrieben, bei welcher der Muttermund detailliert („Transformationszone unauffällig") eingesehen worden sei. Dies sei nur möglich, wenn der Scheideneingang nicht "nahezu zugenäht" worden sei. Ob der häutig hochgezogene Damm aufgrund einer ungünstig platzierten Hautnaht oder durch Wundheilungsstörungen entstanden ist, lasse sich nicht beurteilen, zumal nachträglich eine operative Revision stattgefunden habe, die ihrerseits Wundheilungsstörungen zur Folge gehabt habe. Damit sei die Klägerin für den vor ihr erhobenen Vorwurf eines Behandlungsfehlers beweisfällig geblieben.

\section{Was bedeutet das Urteil für den klinischen Alltag?}

Wieder einmal kam der Dokumentation der einzelnen Akteure entscheidende Bedeutung zu. Auf Grundlage der Dokumentation der Gynäkologin, der Hebamme und des niedergelassenen Kollegen konnte der Vorwurf der Patientin entkräftet werden. Für ein sorgfaltswidriges Vorgehen fand sich kein Hinweis.

Es darf daran erinnert werden, dass die Dokumentation nicht nur mit Sorgfalt zu führen, sondern auch mit Sorgfalt aufzubewahren ist. Die Aufbewahrungsfrist von mindestens zehn Jahren ergibt sich aus dem $\$ 10$ Abs. 3 der Berufsordnung. Im Rahmen des geplanten Patientenrechtsgesetzes ist nach dem

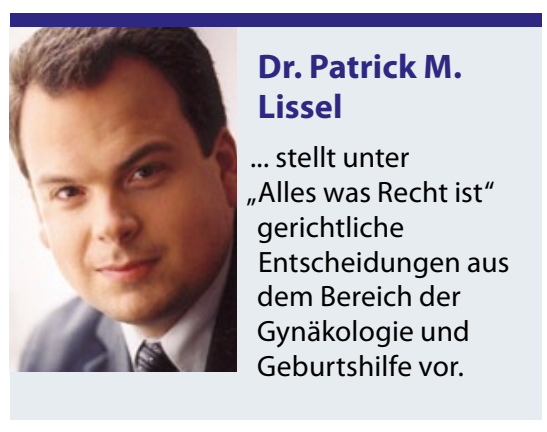

Entwurf der Bundesregierung (vgl. BRDrucks. $312 / 12$ v. 25.5.2012) eine entsprechende Regelung auch im BGB vorgesehen ( $\$$ 630f Abs. 3 BGB-E). Bekanntlich bestehen nach anderen gesetzlichen Bestimmungen längere Aufbewahrungsfristen, beispielsweise nach der Röntgenverordnung oder dem Transfusionsgesetz.

Im oben dargestellten Fall schien die Dokumentation zunächst unauffindbar, und es bestand die Vermutung, dass diese durch eine im Unfrieden ausgeschiedene Mitarbeiterin in Schädigungsabsicht entwendet wurde. Letztendlich stellte sich jedoch heraus, dass die Dokumentation irrtümlich unter der Fallnummer des Kindes archiviert worden war. Die beste Dokumentation nützt also nichts, wenn sie im Falle eines Falles nicht auch vorgelegt werden kann.

\section{Dr. Patrick M. Lissel, LL.M}

Rechtsanwalt und Fachanwalt für Medizinrecht

Ratzel Rechtsanwälte

Ottostraße 1

80333 München 